\title{
Influence de quatre milieux de propagation sur le comportement de six levains lactiques mésophiles du commerce
}

\author{
Renée ROY *, J. GOULET * et P. PROVENCHER ** \\ * Département de Sciences et Technologie des Aliments et Centre de Recherche en Nutrition \\ Université Laval, Québec, Canada G1K 7P4 \\ ** Service des Maladies infectieuses, Centre Hospitalier de l'Université Laval \\ Sainte-Foy, Québec, Canada G1K 7P4
}

\section{Résumé}

Nous avons comparé la performance de six levains lactiques commerciaux dans quatre milieux de propagation : poudres de lait écrémé Lo Heat et Hi Heat et deux milieux phosphatés inhibiteurs de phages. La nature des milieux de culture a influencé le comportement des cultures lactiques en fabrication fromagère simulée. Les différences observées entre les divers milieux sont à l'avantage des poudres Lo Heat et Hi Heat.

Mots clés : Levain - Lait écrémé en poudre - Milieux phosphatés inhibiteurs de phages.

\section{Summary}

Effect of propagation media on the growth and acid production of starter cultures for cheese making

The growth and acid production of six cheddar cheese starter cultures were studied in four propagation media: two heat treated (Lo Heat and Hi Heat) commercial non fat dry milks (NFDM) and two phage-inhibitory media (Nulac and One-2-One).

Highly significant differences in growth and acid production were obtained between NFDM and phage-inhibitory media during propagation; these differences were much less significant upon further transfer into fresh fluid milk (simulated cheese production).

Phage-inhibitory media appeared to enhance the proteolytic activity of most starter cultures under study; this effect did not persist however in the first stage $(4 \mathrm{~h})$ of a simulated cheese production.

Keys words: Starters - Skim milk powder - Phage-inhibitory media. 


\section{Introduction}

Les levains lactiques s'accommodent bien de milieux constitués uniquement de poudre de lait écrémé reconstituée à divers taux d'extraits secs. En raison de leur grande susceptibilité aux phages, on rencontre toutefois dans le commerce divers milieux synthétiques préparés à partir d'une banque de plus de dix-sept ingrédients (SANDiNE, 1977). Surnommés «poudres phosphatées », ces milieux contiennent principalement des extraits de levure, des hydrolysats de caséine, de la poudre de lactosérum, des citrates, du $\mathrm{NaCl}$, du $\mathrm{MgSO}_{4}$, du $\mathrm{FeSO}_{4}$ et divers sels de phosphate. La préparation de tels milieux a fait l'objet d'études d'optimisation (ZotTOla et MARTH, 1966 ; RichaRdson et al., 1977 ; AUSAVANOdOM et al., 1977 ; WRIGHT et RichaRdSON, 1982).

Avec le contrôle externe du $\mathrm{pH}$ en continu, l'activité et la performance des levains ont pu être améliorées considérablement. Par ailleurs, d'après HugGins (1984), la préparation d'un milieu avec contrôle interne du $\mathrm{pH}$ semble donner aussi d'excellents résultats, tant pour l'activité des levains que l'inhibition des phages. Ce dernier avantage disparaît toutefois au moment du transfert de l'inoculum au lait frais de grand mélange.

Nous avons comparé l'utilisation de deux types de poudres de lait (Lo Heat et Hi Heat) ainsi que deux milieux phosphatés commerciaux pour la propagation de six cultures lactiques commerciales à cheddar.

\section{Matériels et méthodes}

\section{A. Préparation des inoculums}

A la suite d'une enquête menée auprès de neuf fromageries québécoises (Goulet et al., 1981), nous avons retenu six levains mixtes commerciaux les plus utilisés pour la production de cheddar : quatre levains concentrés surgelés 91 et 253 (Chr. Hansen's Lab., Ltd., Milwaukee, WI), WP et OS (Miles Lab., division Marschall, Madison, WI) ainsi que deux levains surgelés MAR-3 et N13H conservés en paillettes dans l'azote liquide (Agropur, Granby, Québec).

Chacune des cultures obtenues sous forme de concentré surgelé (en contenant métallique hermétique) était d'abord décongelée dans un bain d'eau chlorée $(200 \mathrm{ppm})$ à $35^{\circ} \mathrm{C}$. Une aliquote du concentré était alors transférée aseptiquement dans le milieu de conservation (poudre de lait Lo Heat, réhydratée à $12 \%$ E.S.T., stérilisée à $121^{\circ} \mathrm{C}, 10 \mathrm{~min}$, et refroidie à $0^{\circ} \mathrm{C}$ ) au taux de $10 \% \mathrm{v} / \mathrm{v}$ (correspondant à une population finale de $10^{8} \mathrm{~b} / \mathrm{mL}$ ). Chaque levain était ensuite réparti en portions de $6 \mathrm{~mL}$ dans des tubes de polypropylène stériles $(17 \mathrm{~mm} \times$ $10 \mathrm{~mm}$, Falcon). Ces derniers étaient gardés à $-80^{\circ} \mathrm{C}$ jusqu'au moment de leur utilisation.

Les deux cultures fournies sous forme de paillettes ont d'abord été cultivées $18 \mathrm{~h}$ à $22^{\circ} \mathrm{C}$ dans du lait autoclavé (poudre de lait écrémé Lo Heat, réhydratée à $12 \%$ E.S.T. et stérilisée à $121^{\circ} \mathrm{C}, 10 \mathrm{~min}$ ) avant d'être transférées dans le 
milieu de conservation à un taux de $15 \% \mathrm{v} / \mathrm{v}$ (correspondant à une population finale de $10^{8} \mathrm{~b} / \mathrm{mL}$ ) et entreposées par la suite à $-80^{\circ} \mathrm{C}$ comme on l'a mentionné.

\section{B. Milieux de culture}

Les essais ont porté sur l'utilisation de deux types de poudre de lait écrémé, la poudre LH (Lo Heat, Aliments Delisle, Boucherville, Québec) et la poudre $\mathrm{HH}$ (Hi Heat, Agrinove, Sainte-Claire, Québec) ainsi que deux milieux phosphatés, la poudre 1:1 (One-2-one, Miles Lab., division Marschall) et NL (Nulac, Miles Lab., division Marschall). Les poudres $\mathrm{LH}$ et $\mathrm{HH}$ ont été reconstituées à $12 \%$ E.S.T. et chauffées à $90^{\circ} \mathrm{C}, 45 \mathrm{~min}$. Les milieux phosphatés ont été préparés selon les recommandations du fournisseur : le milieu $1: 1$ réhydraté à $12 \%$ E.S.T. ( $6 \%$ provenant de la poudre $1: 1$ et $6 \%$ de la poudre $\mathrm{LH})$ a été chauffé à $88^{\circ} \mathrm{C}$ pendant $45 \mathrm{~min}$, alors que la poudre NL réhydratée à $8 \%$ E.S.T. le fut à $90^{\circ} \mathrm{C}$ pendant $45 \mathrm{~min}$. Les milieux de culture ainsi traités ont été refroidis rapidement et gardés $15 \mathrm{~h}$ à $4{ }^{\circ} \mathrm{C}$ avant leur utilisation.

\section{Conditions de culture}

Les fermentations ont été réalisées dans des erlenmeyers stérilisés de $500 \mathrm{~mL}$ contenant $247,5 \mathrm{~mL}$ de milieu de culture tempéré à $22^{\circ} \mathrm{C}$. Une aliquote de $2,5 \mathrm{~mL}$ de levains décongelés $\left(5 \mathrm{~min}\right.$ dans de l'eau à $\left.35^{\circ} \mathrm{C}\right)$ servait d'inoculum. Les erlenmeyers étaient maintenus à $22^{\circ} \mathrm{C}$ dans un bain d'eau, sans agitation, sauf pour le milieu Nulac. Ce dernier était agité continuellement dans une fiole Bellco à fond indenté (Controlled environment incubator shaker, New-Brunswick Scientific, $150 \mathrm{t} / \mathrm{min}$ ) pour prévenir la sédimentation des matières insolubles, précaution suggérée par le fabricant.

La durée d'incubation de chaque culture a été établie d'après le temps nécessaire pour atteindre une acidité titrable de $80^{\circ} \mathrm{D}: 11 \mathrm{~h} 15$ pour les levains 253 et WP, $12 \mathrm{~h} 15$ pour les levains $\mathrm{N} 13 \mathrm{H}$ et $\mathrm{OS}$ et $13 \mathrm{~h} 00$ pour les levains MAR-3 et 91. Au terme de la période d'incubation, ces erlenmeyers étaient refroidis rapidement à $8{ }^{\circ} \mathrm{C}$ puis mis au réfrigérateur $\left(4^{\circ} \mathrm{C}\right)$ pour une période de 12 à $15 \mathrm{~h}$.

Cette étape de propagation d'une durée moyenne de $12 \mathrm{~h}$ était suivie, le lendemain, d'un transfert en lait frais et d'une acidification de $4 \mathrm{~h}$ visant à simuler les premières étapes de la fabrication fromagère. Cette acidification se déroulait aussi dans des erlenmeyers stériles de $500 \mathrm{~mL}$ contenant $247,5 \mathrm{~mL}$ de lait frais entier de grand mélange (3,25\% M.G.), pasteurisé et homogénéisé. Ce lait tempéré à $31^{\circ} \mathrm{C}$ était inoculé au taux de $1 \%$ avec le lait fermenté de la veille $(2,5 \mathrm{~mL})$. Le tout était incubé à $31{ }^{\circ} \mathrm{C}$ pendant $4 \mathrm{~h}$, sans agitation.

\section{Echantillonnage}

Des prélèvements étaient effectués au moment de l'inoculation et au terme de chacune des fermentations pour l'évaluation du $\mathrm{pH}$, de l'acidité titrable, de la population bactérienne viable et de l'acide lactique. 


\section{pH et acidité titrable}

Les mesures du $\mathrm{pH}$ et de l'acidité titrable étaient réalisées au moyen d'un titrimètre automatique (TTT-2 Radiometer, Copenhague, Danemark) dont le point de consigne était ajusté à 8,6 , point de virage de la phénolphtaléine. Une solution $\mathrm{NaOH}$ N/9 était utilisée comme neutralisant.

\section{Cellules viables}

Les dénombrements de cellules viables ont été effectués sur gélose Elliker (Elliker broth $+1,5 \%$ Bacto-agar, Difco) suivant les dilutions décimales appropriées dans de l'eau peptonée $(0,1 \%$ Bacto-peptone, Difco) stérile. Les boîtes de Petri étaient incubées à $22{ }^{\circ} \mathrm{C}, 60 \mathrm{~h}$ avant d'être lues sur compteur automatique (Artek Counter, modèle 880, Artek Systems Corp., N.Y.).

\section{Acide lactique}

Le dosage de l'acide lactique a été effectué selon la méthode de LAWRENCE (1975) à partir d'échantillons dilués $1: 1000$ dans de l'eau désionisée. La courbe étalon a été établie à partir de valeurs situées entre 0 et $10 \mu \mathrm{g}$ de lactate $/ \mathrm{mL}$.

\section{Analyse statistique}

Les essais ont été répétés 2 fois et les résultats obtenus ont fait l'objet d'une analyse de variance de type factoriel à partir des logiciels SAS (Statistical Analysis System, Helwig et Council, 1979) et BMPD (Biomedical Computer Programs, DiXon et Brown, 1979).

\section{Résultats}

L'analyse de variance présentée au tableau I révèle des effets hautement significatifs $(\alpha=0,01)$ des facteurs à l'étude (levains et milieux de propagation) sur les quatre paramètres mesurés (acidité développée, baisse du $\mathrm{pH}$, croissance cellulaire et acide lactique produit). On note aussi des interactions doubles « levains $\times$ milieux » et «milieux à base de lait $(\mathrm{ML}) \times$ milieux phosphatés (MP) ».

En cours de propagation, l'acidification, telle que mesurée par l'acidité développée et l'abaissement du $\mathrm{pH}$ (fig. $1 \mathrm{a}$ et $1 \mathrm{~b}$ ) s'est avérée inférieure dans les milieux phosphatés (1:1 et NL) par rapport aux milieux à base de lait (LH et $\mathrm{HH}$ ). Par ailleurs, certains levains (OS, WP et 253) semblent mieux adaptés que d'autres à croître dans les milieux phosphatés. Ce comportement pourrait expliquer en partie l'interaction «levains $X$ milieux » rapportée plus haut. Nous n'avons pas observé de différence dans la performance de croissance des levains dans les milieux à base de lait; la même observation vaut aussi pour les milieux phosphatés (fig. 1c). Il importe de souligner cependant que des différences non négligeables existent entre les deux groupes de milieux : ceux à base de lait (LH et $\mathrm{HH}$ ) permettent d'obtenir des dénombrements plus élevés pour chacun des levains à l'étude que ceux enrichis en phosphates (1:1 et NL). La quantité d'acide lactique produit est influencée à la fois par les milieux de propagations et les levains (fig. 1d).

En simulation fromagère, les levains préparés dans différents milieux de propagation ont vu leur performance influencée très significativement $(\alpha=0,01)$ 
TABLEAU I

Croissance et activité acidifiante de six levains lactiques dans quatre milieux de propagation $(12 \mathrm{~h})$ : valeurs $F$ obtenues avec analyse de variance Growth and activity of six lactic starters in four growth media : analysis of variance ( $F$ ratio)

\begin{tabular}{|c|c|c|c|c|c|}
\hline Facteur & d. 1. & $\begin{array}{l}\text { Acidité } \\
\text { développée } \\
(\triangle A)\end{array}$ & $\begin{array}{l}\text { Baisse } \\
\text { du } \mathrm{pH} \\
(\Delta \mathrm{pH})\end{array}$ & $\begin{array}{c}\text { Croissance } \\
\text { cellulaire } \\
(\Delta \mathrm{b} / \mathrm{mL})\end{array}$ & $\begin{array}{c}\text { Acide lactique } \\
\text { produit } \\
\text { ( } \Delta \text { lactate) }\end{array}$ \\
\hline Levains & 5 & $103+\pi$ & $65^{\star k}$ & $45 * \pi$ & $11 * \pi$ \\
\hline Milieux (poudres) & 3 & $368 * \pi$ & $3060 * *$ & $120 * \star$ & $29 \div *$ \\
\hline$L H-H H$ & 1 & $32 \star \star$ & $0-$ & $8 * 1$ & $6 *$ \\
\hline$I: I-N L$ & 1 & $129 * \hbar$ & $2-$ & $0-$ & $20 * \pi$ \\
\hline$M L-M P$ & 1 & $942 * \pi$ & $9178 * \pi$ & $353 * \hbar$ & $61 * \pi$ \\
\hline Levains $x$ milieux & 15 & $26 * *$ & $20 * *$ & $5 * *$ & $10 * \frac{\pi}{4}$ \\
\hline
\end{tabular}

Légende:

* significatif a $\alpha=0,01$
* significatif a $\alpha=0,05$
- non significatif

LH $=$ poudre lo-heat

$\mathrm{HH}=$ poudre hi-heat

NL $=$ Nulac

$M L=$ poudres de lait

MP = poudres phosphatées

\section{TABLEAU II}

Croissance et activité acidifiante de six levains lactiques

(cultivés dans quatre milieux de propagation différents)

dans un lait frais de grand mélange $(4 \mathrm{~h})$ : valeurs $F$ obtenues avec analyse de variance

Growth and activity of six lactic starters (grown on four different media) in bulk raw milk : analysis of variance ( $F$ ratio)

\begin{tabular}{|c|c|c|c|c|c|}
\hline Facteur & d. 1 . & $\begin{array}{c}\text { Acidité } \\
\text { développée } \\
(\Delta A)\end{array}$ & $\begin{array}{l}\text { Baisse } \\
\text { du } \mathrm{pH} \\
(\Delta \mathrm{pH})\end{array}$ & $\begin{array}{c}\text { Croissance } \\
\text { cellulaire } \\
(\Delta b / \mathrm{mL})\end{array}$ & $\begin{array}{c}\text { Acide lactique } \\
\text { produit } \\
\text { (Alactate) }\end{array}$ \\
\hline Levains & 5 & $48 * *$ & $70 * *$ & $55 * *$ & $58 * \pi$ \\
\hline Mili eux & 3 & $101 * \hbar$ & $162 * \pi$ & $55 * \pi$ & $51 * *$ \\
\hline$L H-H H$ & 1 & $148 * \hbar$ & $340 * *$ & $143 * *$ & $46 * \pi$ \\
\hline $1: 1-N L$ & 1 & $153 * *$ & $143 * *$ & $8-$ & $105^{* *}$ \\
\hline$M L-M P$ & 1 & $2-$ & $3-$ & $14 * \pi$ & $2-$ \\
\hline Levains $x$ milieux & 15 & $15 * *$ & $16 * \pi$ & $9 \div *$ & $9 * x$ \\
\hline
\end{tabular}

par leur nature propre et celle des milieux dans lesquels ils ont été propagés (tabl. II) ; une interaction double « levains $\times$ milieux » a aussi été observée.

Deux cultures se distinguent des autres par leur performance en production fromagère simulée, ce sont les levains WP et OS sur milieux LH et NL (fig. 2). Avec 

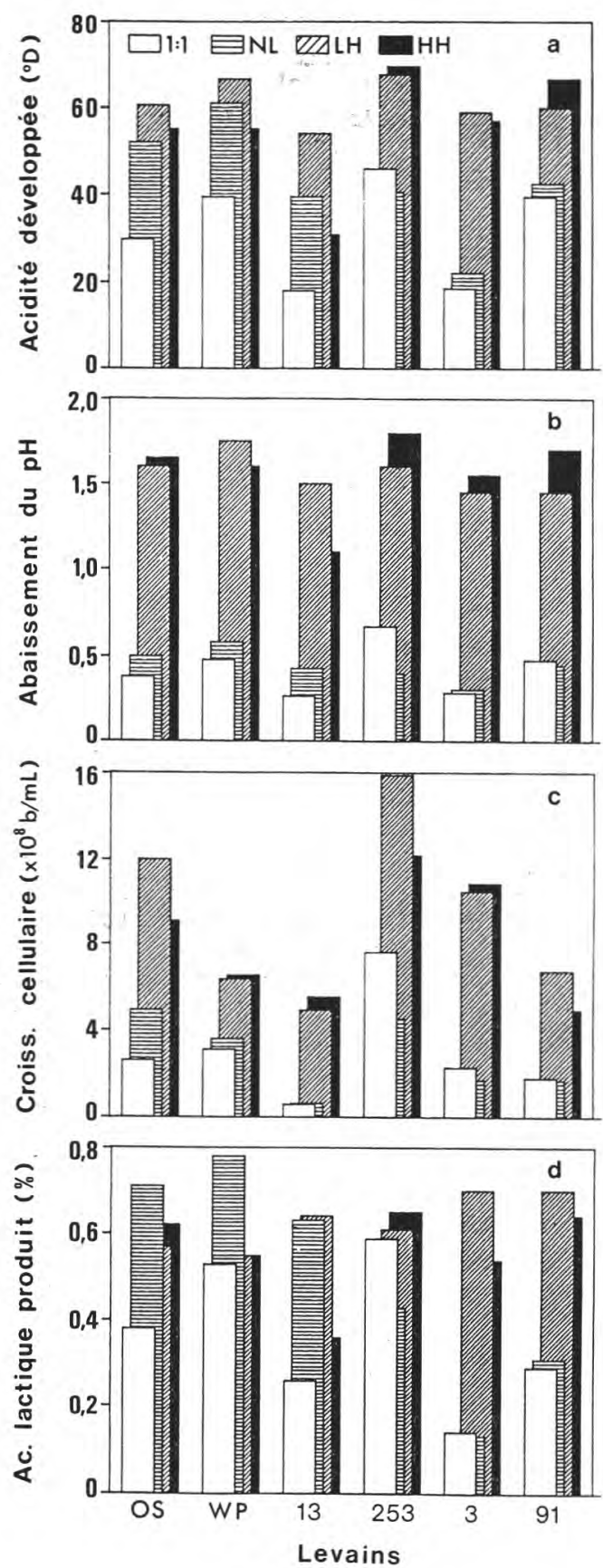

Fig. 1

Comportement de six levains lactiques dans quatre milieux de propagation $(12 \mathrm{~h})$ : acidification $(a)$; abaissement $d u p H(b)$; croissance cellulaire $(c)$; production d'acide lactique (d).

Performance of six lactic starters in four growth media $(12 \mathrm{~h})$ : acidification (a) ; $p H$ decrease $(b)$; cell growth $(c)$; lactic acid production (d). 

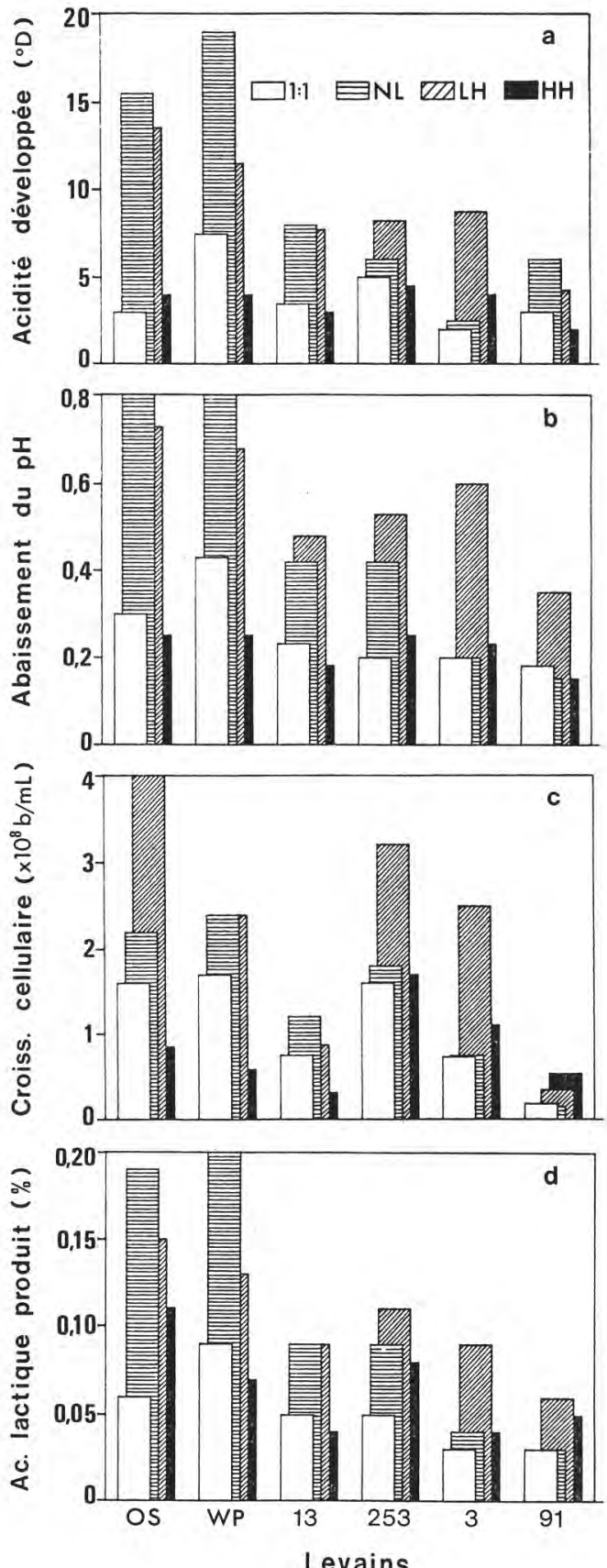

Fig. 2

Comportement de six levains lactiques (cultivés dans quatre milieux de propagation différents) dans un lait frais de grand mélange $(4 \mathrm{~h})$ : acidification $(a)$; abaissement du $p H(b)$; croissance cellulaire (c) ; production d'acide lactique (d).

Performance of six lactic starters (grown on four different media) in bulk raw milk $(4 \mathrm{~h})$ : acidification $(a) ; p H$ decrease $(b)$; cell growth $(c)$; lactic acid production $(d)$. 
ces cultures, nous avons obtenu des acidifications (acidité développée et abaissement du $\mathrm{pH}$ ) nettement supérieures aux autres levains sur les deux milieux mentionnés (fig. $2 \mathrm{a}$ et $2 \mathrm{~b}$ ). Les résultats présentés à la figure $2 \mathrm{~d}$ font ressortir une forte production d'acide lactique par les levains WP et OS préalablement cultivés sur milieux LH et NL. Les autres cultures produisent du lactate en quantité inférieure indépendamment du milieu de propagation utilisé.

\section{Discussion et conclusion}

Les résultats de cette étude permettent d'avancer que la nature des milieux utilisés pour la propagation des levains lactiques conditionne le comportement de ces derniers en début de fabrication fromagère. L'analyse de variance a révélé un effet hautement significatif $(\alpha=0,01)$ du type de milieu de propagation sur les caractéristiques de croissance et d'acidification des levains tant dans le milieu de propagation lui-même que lors du transfert en lait frais dans une production fromagère simulée. Des différences hautement significatives existent aussi entre les levains commerciaux quant à leur comportement dans des conditions identiques de propagation et de démarrage d'une fermentation fromagère. Les interactions observées tiennent probablement au fait que tous les levains utilisés ne se comportent pas de la même façon dans les mêmes milieux, certains y étant mieux adaptés que d'autres.

A l'instar de Gulstrom et al. (1979) et de Ledford et SPECK (1979), nous avons observé que certaines souches croissent mieux que d'autres dans les milieux phosphatés, dits inhibiteurs de phages ; l'effet inhibiteur des hautes concentrations de sels est moins prononcé lorsque les souches et le milieu phosphaté sont de même source commerciale. En effet, les levains WP et OS propagés sur milieu NL se sont mieux comportés en démarrage fromager que lorsque préparés sur milieu à base de poudre LH. Par ailleurs, le milieu $1: 1$ ne semble favoriser spécifiquement la croissance d'aucun des levains sélectionnés.

Tel que mis en évidence par les données de la figure $1 \mathrm{~b}$, les poudres phosphatées ont un pouvoir tampon nettement plus élevé que les milieux de propagation conventionnels à base de poudre de lait. Cette particularité n'arrive cependant pas à expliquer l'inconsistance apparente existant entre les pourcentages d'acide lactique mesurés dans les milieux $1: 1$ et NL (fig. 1d) et l'acidité développée dans les mêmes milieux (fig. 1a). En effet, pour les ferments OS, WP, 13 et 253 , la quantité d'acide lactique produite est supérieure à l'acidité développée telle que mesurée par l'acidité titrable, alors que pour les autres ferments il y a une bonne concordance entre les deux valeurs.

A la lumière des dénombrements cellulaires rapportés à la figure $1 \mathrm{c}$, un tel comportement ne semble pas explicable par des différences majeures dans la croissance bactérienne. Les résultats d'acidification (fig. 1a et 1d) nous permettent cependant de postuler une activité protéolytique supérieure des ferments OS, WP, 13 et 253 dans les milieux phosphatés : les acides aminés et peptides issus 
de la protéolyse auraient contribué à neutraliser une partie de l'acide lactique produit.

Un tel effet stimulateur sur la protéolyse pourrait-il être lié à la séquestration du calcium dans les milieux de propagation enrichis en phosphates? Une telle hypothèse irait à l'encontre des observations d'EXTERKATE (1979) associant l'accumulation d'activité protéinasique dans la paroi cellulaire de Streptococcus cremoris à la présence d'ions $\mathrm{Ca}^{++}$dans le milieu. Par ailleurs, HugenHoltz et al . (1984) ont nuancé cette observation en rapportant une absence de protéolyse chez quelques souches de $S$. cremoris cultivées en milieu MRS enrichi en calcium mais appauvri en phosphates.

Nos données suggéreraient qu'un enrichissement du milieu en phosphates, tel que pratiqué commercialement dans la production de milieux anti-phages, pourrait stimuler la protéolyse chez plusieurs souches de $S$. cremoris. Cette stimulation disparaîtrait cependant lors du transfert des ferments en lait frais : c'est du moins la conclusion qui se dégage en comparant les résultats de la figure $2 \mathrm{~d}$ à ceux de la figure $2 \mathrm{a}$.

Il convient de signaler enfin le piètre comportement de toutes les cultures propagées sur milieu $\mathrm{HH}$ lors du transfert en lait frais. Des travaux ultérieurs n'ont pas laissé voir de différence aussi marquée dans le comportement des levains cultivés sur milieu LH ou HH. Soulignons que, dans le cadre du protocole expérimental suivi, la qualité du lait de grand mélange utilisé en simulation fromagère pouvait varier d'une semaine à l'autre. Puisque nos essais sur chaque type de milieu de culture ont été concentrés à l'intérieur d'une même semaine, le comportement observé peut n'être qu'accidentel. La variabilité des laits de grand mélange d'une journée, d'une semaine et d'une saison à l'autre, rend difficile des comparaisons aussi subtiles. Soulignons qu'en dépit des variations de tout ordre auxquelles les fromagers sont quotidiennement confrontés, ils réussissent généralement leurs fabrications de fromage à taux relativement élevé.

Les résultats de notre étude permettent de conclure que le bon comportement d'un levain dans un milieu de propagation n'est pas toujours synonyme d'un bon comportement dans un lait frais de grand mélange, même lorsque ce dernier est exempt d'agents inhibiteurs détectables : la présence de phosphates dans les milieux de propagation semble avoir un effet non négligeable sur l'activité des ferments destinés à la fabrication fromagère.

\section{Remerciements}

Cette recherche a été réalisée grâce à un contrat de la Commission canadienne du lait (OSU81-00310). Nous tenons à remercier le Dr D.A. Emmons (Agriculture Canada) pour ses précieux conseils. 


\section{Références bibliographiques}

Ausavanodom N., White R.S., Young G., Richardson G.H., 1977. Lactic bulk culture system utilizing whey-based bacteriophage inhibitory medium and $\mathrm{pH}$ control. II. Reduction of phosphate requirements under $\mathrm{pH}$ control. J. Dairy Sci., 60, 1245-1251.

DIXoN W.J., BRown M.B., 1979. BMDP Biochemical computer programs $P$ series. Univ. California Press, Los Angeles.

EXTERKATE F.A., 1979. Accumulation of proteinase in the cell wall of Streptococcus cremoris strain $\mathrm{AM}_{1}$ and regulation of its production. Arch. Microbiol., 120, $247-254$.

Goulet J., RoY R., SAINT-ClaIR P.M., 1981. Ferments lactiques et production fromagère : importance de la régie. Contrat OSU80-00335, Commission canadienne du lait, Agriculture Canada.

Gulstrom T.J., Pearce L.E., SANDine W.E., Elliker P.R., 1979. Evaluation of commercial phage inhibitory media. J. Dairy Sci., 62, 208-221.

Helwig J.T., CouncIl K.A., 1979. SAS user's guide 1979 edition. Cary North Carolina, 494 p.

Hugenholtz J., EXTERKate F., Konings W.N., 1984. The proteolytic systems of Streptococcus cremoris : an immunological analysis. Appl. Environ. Microbiol., 48, 1105-1110.

HugGins A.R., 1984. Progress in dairy starter culture technology. Food Technol., 38, 41-50.

LAWRENCE A.J., 1975. Determination of lactic acid in cream. Aust. J. Dairy Technol., 30, 14-15.

LEDFORD R.A., SPECK M.L., 1979. Injury of lactic streptococci by culturing in media containing high phosphate. J. Dairy Sci., 62, 781-784.

Richardson G.H., CHENG C.T., Young R., 1977. Lactic bulk culture system utilizing a wheybased bacteriophage inhibitory medium and $\mathrm{pH}$ control. I. Applicability to American style cheese. J. Dairy Sci., 60, 378-386.

SANDINE W.E., 1977. New techniques in handling lactic cultures to enhance their performance. J. Dairy Sci., 60, 822-828.

WRIGHT S.L., RICHARDSON G.H., 1982. Optimization of whey-based or nonfat dry milk-based media for production of $\mathrm{pH}$ controlled bulk lactic cultures. J. Dairy Sci., 65, 1882-1889.

ZotTola E.A., MaRth E.H., 1966. Dry-blended phosphate-treated milk media for inhibition of bacteriophages active against lactic strepococci. J. Dairy Sci., 49, 1343-1349. 